\title{
Tratamento clínico do linfangioma com alfa-2a-interferon
}

\author{
Treatment of lymphangioma with alpha-2a-interferon
}

\author{
Reynaldo J.S.P. de Souza ${ }^{1}$, Luiz G. Tone ${ }^{2}$
}

\section{Resumo}

Objetivo: descrever os resultados obtidos com o uso do alfa-2ainterferon no tratamento de pacientes pediátricos com linfangiomas irressecáveis ou refratários a outros tratamentos.

Método: após revisão da literatura sobre a patogenia e os tratamentos disponíveis para os linfangiomas, utilizamos o alfa-2ainterferon na dosagem de 3 milhões de unidades $/ \mathrm{m}^{2} / \mathrm{dia}$, via subcutânea, para tratamento de pacientes que apresentavam lesões irressecáveis. Para avaliar o grau de resposta e os efeitos colaterais foram realizadas avaliações clínicas e laboratoriais periódicas.

Resultados: observamos que os 6 pacientes com linfangiomas irressecáveis que utilizaram alfa-2a-interferon obtiveram uma resposta clínica satisfatória associada a efeitos colaterais mínimos. Cinco destes evoluíram com regressão parcial de suas lesões e 1 com estabilização da doença.

Conclusão: concluímos que o alfa-2a-interferon constitui uma das armas disponíveis para o tratamento dos linfangiomas inoperáveis na infância.

J Pediatr (Rio J) 2001; 77 (2): 139-42: linfangioma, higroma cístico, interferon.

\section{Introdução}

A definição de linfangioma, primeiramente descrito em 1828 por Redenbacher (apud Martinot, 1997) ${ }^{1}$, permanece controversa. Alguns autores o consideram uma malformação congênita do sistema linfático ${ }^{2-5}$, enquanto outros um hamartoma congênito ${ }^{3,6}$ ou um tumor vascular benigno decorrente da proliferação de vasos linfáticos ${ }^{7}$.

A sua ocorrência é relativamente rara $^{1,6,8}$, sendo observado principalmente nos dois primeiros anos de vida. A

1 Médico Pediatra, especialização em Oncologia/Hematologia Pediátrica pelo Hospital das Clínicas da Faculdade de Medicina de Ribeirão Preto da Universidade de São Paulo.

2 Professor Assistente Doutor do Departamento de Puericultura e Pediatria do Hospital das Clínicas da Faculdade de Medicina de Ribeirão Preto da Universidade de São Paulo.

\begin{abstract}
Objective: to describe the results of the use of alpha-2a-interferon in the treatment of inoperable childhood lymphangiomas refractory to other therapeutic management.

Method: we reviewed the literature about pathogenic events and the treatments available for lymphangiomas. Based on that, we used alpha-2a-interferon at a dose of 3 million units $/ \mathrm{m}^{2} /$ day administered subcutaneously to patients with inoperable disease and no response to other treatments. We conducted periodic clinical and laboratory control to evaluate the response and the adverse reactions to alpha2a-interferon administration.

Results: we observed that the 6 patients with inoperable lymphangiomas who used alpha-2a-interferon had a satisfactory clinical response associated with minimum adverse reactions. Of these patients, 5 had partial regression of their lesions, and 1 remained stable.

Conclusion: alpha-2a-interferon may be one more available treatment for inoperable childhood lymphangiomas.
\end{abstract}

J Pediatr (Rio J) 2001; 77 (2): 139-42: lymphangioma, cystic hygroma, interferon.

região da cabeça e do pescoço permanece a mais freqüentemente comprometida, seguida pela extremidade, tronco e abdômen ${ }^{3,4,7-9}$. Normalmente esta lesão é assintomática, tornando-se evidente como uma massa cística, indolor, aderida aos planos profundos, recoberta por pele normal, com crescimento lento e tamanho flutuante.

Embora histologicamente benigno, devido ao seu caráter infiltrativo, o linfangioma pode expandir para dentro dos tecidos adjacentes e/ou estruturas vitais, causando complicações, algumas ameaçadoras à vida ${ }^{1,2,4,5,8,9}$. Há relatos de regressão espontânea, porém a sua ocorrência não é comum.

O envolvimento da língua é extremamente raro, podendo ser localizado ou difuso, sendo esse último a maioria dos 
casos. A língua assume uma aparência granular com múltiplos cistos transparentes preenchidos por linfa, ocasionalmente podendo ocorrer sangramento dentro destas vesículas, tornando-as hemorrágicas. A língua, aumentada de tamanho, usualmente apresenta-se protusa, seca e com fissuras, o que pode dificultar a mastigação, deglutição e linguagem, além de causar anormalidades ortodônticas e distúrbios psicológicos ${ }^{3}$.

Vários métodos de tratamento têm sido propostos, dentre eles aspiração, incisão e drenagem (podem ter um papel importante na descompressão de emergência, não sendo considerado um tratamento definitivo) ${ }^{8}$, esteróides, laser, quimioterapia, irradiação, esclerose química com bleomicina, glicose 50\%, doxiciclina, Ethibloc ${ }^{\circledR}$ ou OK-432 e alfa-2a-interferon (a-2a-INF). Alguns desses apresentam resultados insatisfatórios e complicações inaceitáveis ${ }^{3,7}$.

A ressecção cirúrgica completa tem sido o tratamento de preferência ${ }^{3,5-7,9}$, porém, pelo caráter infiltrativo do linfangioma, que torna esse procedimento difícil e incompleto, é possível em apenas $10 \%$ a $50 \%$ dos $\operatorname{casos}^{6,4,7-9}$. Devido a isso, a recorrência é um problema importante, variando sua taxa de incidência entre $0 \%$ e $27 \%$ após exérese total e entre $15 \%$ e $53 \%$ após exérese parcial ${ }^{4,8,9}$. As complicações cirúrgicas, que ocorrem em 19\% a 33\% dos $\operatorname{casos}^{8}$, incluem formação de hematoma, linfocele, cicatriz, abcesso, infecção, deiscência de sutura e paralisia nervosa ${ }^{1,8,9}$.

Dentre os agentes esclerosantes destaca-se o OK-432, uma mistura liofilizada da proteína Su do Streptococcus pyogenes do grupo $\mathrm{A}$, incubada com penicilina $\mathrm{G}$, que teve seu uso no tratamento dos linfangiomas inicialmente relatado por Ogita e cols., em $1987^{10}$. Essa droga obteve uma taxa de resposta de até $92 \%$ com efeitos colaterais mínimos e nenhum dano cicatricial à pele ${ }^{2,6,9}$. Até o momento não está disponível comercialmente no Brasil.

Através deste artigo, relatamos nossa experiência com o uso do a-2a-INF no tratamento dos linfangiomas progressivos e irressecáveis.

\section{Relato dos Casos}

Relatamos 6 casos de pacientes com idades entre 23 dias e 17 anos, 4 do sexo masculino e 2 do sexo feminino, que receberam a-2a-INF, todos com linfangiomas recorrentes, irressecáveis e progressivos (Tabela 1).

Uma vez confirmado o diagnóstico através de avaliação clínica, de imagem e/ou biópsia, os pacientes foram classificados de acordo com a proposta de Landing e Farber, que divide os linfangiomas em três tipos anatômicos: a) linfangioma capilar ou circunscrito, correspondendo a vesículas cutâneas de conteúdo claro; b) linfangioma cavernoso, uma massa cutânea ou subcutânea maldefinida e c) linfangioma cístico macrocístico - quando existe uma ou várias cavidades suficientemente grandes permitindo sua visualização ecocardiogáfica - e microcístico - com cavidades puntiformes (apud Balakrishnan, 1991) ${ }^{3}$. Os que apresentavam lesões irressecáveis iniciaram o a-2a-INF, na dosagem de $3.000 .000 \mathrm{U} / \mathrm{m}^{2} / \mathrm{dia}$, via subcutânea, diariamente. Apenas em um paciente essa droga foi administrada $3 \mathrm{vezes} / \mathrm{sema}$ na, pois ainda não tinhamos definido o esquema de utilização do a-2a-INF. Aqueles com lesões hemangiomatosas associadas receberam ainda prednisona e ácido épsilonaminocapróico. Durante todo o período de uso do a-2aINF, os pacientes realizaram periodicamente exames hematológicos, bioquímicos e avaliação oftalmológica de controle.

Os critérios de resposta utilizados estavam de acordo com a Organização Mundial de Saúde e foram aplicados sempre que fossem possíveis: RC (resposta completa), desaparecimento de toda lesão conhecida por pelo menos 4 semanas; RP (resposta parcial), diminuição de $50 \%$ ou mais da lesão por pelo menos 4 semanas, sem progressão de qualquer lesão tumoral e nenhuma ocorrência de lesões novas; DE (doença estável), menos de 50\% de diminuição e menos que $25 \%$ de aumento, e nenhuma lesão nova; DP (doença progressiva), aumento de $25 \%$ ou mais, ou surgimento de novas lesões ${ }^{11}$.

\section{Discussão}

$\mathrm{O}$ alfa-2a-interferon tem sido utilizado com ótimos resultados no tratamento dos hemangiomas ${ }^{7,10-15}$. Isso levou Reinhardt e cols. (1997) a examinarem a sua efetividade no tratamento de dois pacientes com linfangioma irressecável, associado a derrame pleural. Ambos obtiveram boa resposta com melhora da sintomatologia e diminuição do número de internações requeridas, com efeitos colaterais mínimos ${ }^{7}$.

In vitro, o a-2a-INF atua de forma direta, inibindo a migração e proliferações de células endoteliais e outros passos da angiogênese. Estudos in vivo, em modelos animais, com linfócitos e angiogênese induzida por células tumorais, o a-2a-INF demonstrou também atuar indiretamente, inibindo o estímulo angiogênico, através da inibição dos efeitos de fatores de crescimento específicos sobre a proliferação de células endoteliais ${ }^{12,13}$.

Maddalozzo e cols. (1999), baseados na evidência de que os higromas císticos, também chamados de linfangiomas císticos, são caracterizados por uma proliferação de pequenos vasos sangüíneos e linfáticos, e de que alguns tumores malignos e benignos são dependentes de um desequilíbrio no balanço de indutores e inibidores da angiogênese, postularam que as células do higroma cístico são angiogênicas devido à secreção de altos níveis de indutores angiogênicos que promovem sua proliferação. Em seus estudos, demostraram, in vitro, que a atividade angiogênica das células do higroma cístico era devida à secreção de altos níveis de BFGF (basic fibroblast growth factor), um indutor angiogênico, e baixos níveis de TSP-1 (trombospondin1), um inibidor angiogênico. Com isso concluíram que o linfangioma pode representar uma neoplasia dependente da angiogênese ${ }^{15}$. 
Tabela 1 - Características clínicas e dos tratamentos dos pacientes com linfangiomas

\begin{tabular}{|c|c|c|c|c|c|c|c|c|c|}
\hline $\begin{array}{l}\text { Pet. } \\
\text { n }^{\mathbf{0}}\end{array}$ & Idade & $\begin{array}{l}\text { Sexo } \\
\text { Raça }\end{array}$ & $\begin{array}{l}\text { Localização } \\
\text { da lesão }\end{array}$ & $\begin{array}{c}\text { Classifi- } \\
\text { cação do } \\
\text { lingangioma }\end{array}$ & $\begin{array}{c}\text { Sinais e/ou } \\
\text { sintomas } \\
\text { associados }\end{array}$ & Tratamentos & $\begin{array}{c}\text { Intercorrências } \\
\text { decorrentes } \\
\text { do tratamento }\end{array}$ & $\begin{array}{c}\text { Duração } \\
\text { do tra- } \\
\text { tamento }\end{array}$ & $\begin{array}{r}\text { Resposta ao } \\
\text { tratamento } \\
\text { empregado }\end{array}$ \\
\hline 1 & $8 \mathrm{a} 11 \mathrm{~m}$ & $\mathrm{M} / \mathrm{B}$ & $\begin{array}{l}\text { Cervical direita } \\
\text { Língua e lábio }\end{array}$ & $\begin{array}{l}\text { Cavernoso } \\
\text { Capilar }\end{array}$ & - & $\begin{array}{l}2 \text { Cirurgias } \dagger \\
\text { INF } 3.000 .000 \mathrm{u} / \mathrm{m}^{2} / \mathrm{d}^{*}\end{array}$ & $\begin{array}{l}\text { Paresia da língua, } \\
\text { paralisia do lábio } \\
\text { inferior } \\
\text { - }\end{array}$ & $11 \mathrm{~m}$ & $\mathrm{RP}$ \\
\hline 2 & $17 \mathrm{a}$ & $\mathrm{F} / \mathrm{B}$ & Língua & Capilar & - & $\begin{array}{l}6 \text { Cirurgias } \\
\text { INF } 3.000 .000 \mathrm{u} / \mathrm{m}^{2} \\
3 \mathrm{x} / \mathrm{sem}^{*}\end{array}$ & $\begin{array}{l}\text { Dislalia } \\
\text { Febre } 38^{\circ} \mathrm{C} \text { na } \\
1^{\text {a }} \text { semana }\end{array}$ & $7 \mathrm{~m}$ & $\begin{array}{l}\mathrm{DP} \\
\mathrm{RP}\end{array}$ \\
\hline 3 & $23 d$ & $\mathrm{M} / \mathrm{B}$ & Parede torácica & Macrocístico & - & INF $3.000 .000 \mathrm{u} / \mathrm{m}^{2} / \mathrm{d}$ & $\begin{array}{l}\text { Febre } 37,8^{\circ} \mathrm{Ce} \\
\text { irritação na } \\
1^{\mathrm{a}} \text { semana }\end{array}$ & $6 \mathrm{~m}$ & $\mathrm{RP}$ \\
\hline 4 & $2 \mathrm{~m}$ & $\mathrm{~F} / \mathrm{B}$ & $\begin{array}{l}\text { Sublingual } \\
\text { Cervical }\end{array}$ & $\begin{array}{l}\text { Capilar } \\
\text { Cavernoso }\end{array}$ & $\begin{array}{c}\text { Compressão } \\
\text { de VAS }\end{array}$ & $\begin{array}{l}\text { INF } 3.000 .000 \mathrm{u} / \mathrm{m}^{2} / \mathrm{d} \\
\text { OK432** }\end{array}$ & $\begin{array}{l}- \\
\text { Febre } 38^{\circ} \mathrm{C} \text {, reação } \\
\text { inflamatória local } \\
\text { na } 1^{\mathrm{a}} \text { semana }\end{array}$ & $\begin{array}{l}2 \mathrm{~m} \\
2 \text { apli- } \\
\text { cações }\end{array}$ & $\begin{array}{l}\mathrm{DE} \\
\mathrm{RP}\end{array}$ \\
\hline 5 & $11 \mathrm{a} 10 \mathrm{~m}$ & $\mathrm{M} / \mathrm{P}$ & $\begin{array}{l}\text { Sublingual, } \\
\text { Cervical bilateral } \\
\text { e submandibular }\end{array}$ & $\begin{array}{l}\text { Capilar } \\
\text { Cavernoso }\end{array}$ & & $\begin{array}{l}2 \text { cirurgias } \dagger \\
\text { INF } 3.000 .000 \mathrm{u} / \mathrm{m}^{2} / \mathrm{d}\end{array}$ & $\begin{array}{l}\text { - } \\
\text { Febre } 38^{\circ} \mathrm{C} \text { por } 4 \mathrm{~d} \\
\text { e cansaço por } 10 \mathrm{~d} \text {. } \\
\text { Aumento TGO } \\
\text { e TGP }\end{array}$ & $7 \mathrm{~m}$ & $\begin{array}{c}\text { DP } \\
\text { DP } \\
\text { DP RP }\end{array}$ \\
\hline 6 & $3 a$ & $\mathrm{M} / \mathrm{N}$ & $\begin{array}{l}\text { Auricular, cer- } \\
\text { vical, subman- } \\
\text { dibular, supra- } \\
\text { clavicular es- } \\
\text { querda, } 1 / 3 \text { su- } \\
\text { perior do me- } \\
\text { diastino anterior }\end{array}$ & Macrocístico & $\begin{array}{c}\text { Compressão } \\
\text { de VAS, } \\
\text { dislalia, } \\
\text { distúrbios } \\
\text { psicológicos }\end{array}$ & $\begin{array}{l}2 \text { cirurgias } \\
\text { INF } 3.000 .000 \mathrm{u} / \mathrm{m}^{2} / \mathrm{d}\end{array}$ & $\begin{array}{l}\text { PCR, abcesso, lin- } \\
\text { focele, paralisia } \\
\text { facial esquerda } \\
\text { Febre } 38^{\circ} \mathrm{Ce} \\
\text { cansaço na } \\
1^{\text {a }} \text { semana }\end{array}$ & $1 \mathrm{a} 4 \mathrm{~m}$ & DP \\
\hline
\end{tabular}

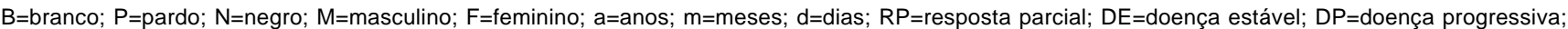
VAS=vias aéreas superiores; $P C R=$ parada cardiorrespiratória

† 1 cirurgia na região cervical e 1 na língua

* uso concomitante com ácido épsilon-aminocapróico e prednisona, paciente o 1 por 7 e 4 meses respectivamente; paciente no 2 por 1 mês

** Aplicado em 2 seções após DE com o uso do a-2a-Interferon

Dos pacientes aqui descritos, 6 utilizaram e/ou estão utilizando a-2a-INF. Os efeitos colaterais mais comumente observados foram febre (de até $38^{\circ} \mathrm{C}$ ), anorexia e cansaço que desapareceram após alguns dias do início do tratamento e não justificaram qualquer alteração na administração da droga. Nas avaliações hematológicas de controle, nenhum paciente apresentou leucopenia e/ou trombocitopenia importante; contudo, na avaliação bioquímica, o paciente 5 cursou com um aumento das transaminases após um mês de uso do a-2a-INF (pré-tratamento, TGO 25,0 UI/l e TGP 34,5 UI/l; controle, TGO 134,7 UI/l e TGP 160,2 UI/l), tendo-se então optado por realizar a medicação em dias alternados até retorno dessas enzimas aos seus níveis basais, o que ocorreu no prazo de 15 dias.

Dos pacientes que receberam a-2a-INF, apenas o paciente 4 não apresentou diminuição nas dimensões de sua lesão. Não podemos inferir que isso se deva a uma falha terapêutica, visto que a lesão também não progrediu, assim como não podemos descartar a possibilidade do a-2a-INF ter cessado ou diminuído a velocidade de progressão da doença, sendo sua resposta classificada como DE. Como complementação do seu tratamento, foram realizadas duas aplicações do OK-432 com boa resposta. Todos os 5 pacientes restantes apresentaram uma resposta parcial. Dentre esses, o paciente 5 cursou com o desaparecimento de todas as lesões, exceto a sublingual. Após o primeiro mês de tratamento, porém, com a modificação do a-2a-INF para dias alternados devido à elevação das transaminases, essas lesões recidivaram em 15 dias. No momento esse paciente encontra-se em uso diário do a-2a-INF e apresenta apenas a lesão sublingual e nenhuma alteração nas transaminases. O paciente 6 evoluiu com desaparecimento da infiltração 
mediastinal e melhora do grau de compressão das vias aéreas superiores, diminuindo dessa forma a dificuldade respiratória apresentada inicialmente.

Os pacientes 1 e 2 receberam ainda prednisona e ácido épsilon-aminocapróico. $\mathrm{O}$ uso dessas drogas baseou-se na evidência de lesões hemangiomatosas associadas. A prednisona, muito utilizada no tratamento dos hemangiomas, atuaria na angiogênese como um esteróide angiostático, bloqueando os receptores de estrogênio anormalmente aumentados nos hemangiomas e inibindo fatores angiogêni$\cos ^{16,17}$. Isso poderia trazer alguma vantagem aos nossos pacientes, tanto no que diz respeito às lesões hemangiomatosas quanto às lesões linfangiomatosas. $\mathrm{O}$ ácido épsilonaminocapróico, interferindo no mecanismo fibrinolítico, cuja ativação dentro dos hemangiomas preveniria a ocorrência de trombose, evitaria a dissolução de trombos formados na microcirculação dos hemangiomas ${ }^{18,19}$. Como os pacientes em questão não apresentaram nenhuma vantagem aparente sobre aqueles em uso apenas do a-2a-INF, essas drogas foram suspensas.

Com base no mecanismo de ação do a-2a-INF nas lesões angiogênicas, nos resultados obtidos por Reinhandt e cols. ${ }^{7}$ utilizando a-2a-INF em dois pacientes com linfangioma, nos recentes resultados de Maddalozzo e cols ${ }^{15}$. demonstrando uma atividade angiogênica no linfangioma, e nos resultados obtidos com o uso de a-2a-INF em nossos pacientes (embora nenhum deles tenha obtido uma resposta completa), supomos que essa droga tenha um papel importante no tratamento destas lesões, com efeitos colaterais mínimos, e certamente poderá ser utilizada como uma das armas disponíveis na terapêutica dos linfangiomas, principalmente nos pacientes com lesões irressecáveis ou ameaçadoras à vida ou cuja cirurgia possa ser mutiladora.

É importante ressaltar que na maioria dos nossos pacientes a resposta ao tratamento se deu de maneira lenta e gradativa. A falta de resposta nas primeiras semanas de uso da medicação pode não significar uma falha terapêutica. A avaliação do grau de resposta ao tratamento deve ser feita com cuidado, pois as lesões multicísticas, nas quais esses cistos são comunicantes, podem sofrer variações nas suas dimensões devido à passagem de quantidades variáveis do conteúdo de um cisto para o outro.

Muitas questões permanecem sem resposta: qual a posologia ideal, a duração do tratamento, o tipo anatômico com melhor resposta, o mecanismo de ação do a-2a-INF. Sendo assim, vários estudos ainda são necessários para confirmar ou não as nossas observações.

\section{Referências bibliográficas}

1. Martinot V, Descamps S, Février P, Patenotre P, Brevière JM, Piette F, et al. Évaluation du tratement des lymphangiomes kystiques par injections percutanée d'Éthibloc ${ }^{\circledR}$ chez 20 patients. Arch Pédiatr 1997; 4: 8-14.
2. Ogita S, Tsuto T, Nakamura K, Deguchi E, Iwai N. OK-432 therapy in 64 patients with lymphangioma. J Pediatr Surg 1994; 29: 784-5.

3. Balakrishnan A, Bailey CM. Lymphangioma of the tongue. A review of pathogenesis, treatment and the use of surface laser photocoagulation. J Laryng Otol 1991; 105: 924-30.

4. Lille ST, Rand RP, Tapper D, Gruss JS. The surgical management of giant cervicofacial lymphatic malformations. J Pediatr Surg 1996; 31: 1648-50.

5. Molitch HI, Unger EC, Witte CL, VanSonnenberg E. Percutaneous sclerotherapy of lymphangiomas. Radiology 1995; 194: 343-7.

6. Smiths RJH, Burke DK, Sato Y, Poust RI, Kimura K, Bauman NM. OK-432 therapy for lymphangiomas. Arch Otolaryngol Head Neck Surg 1996; 122: 1195-9.

7. Reinhardt MA, Nelson SC, Sencer SF, Bostrom BC, Kurachek SC, Nesbit ME. Treatment of childhood lymphangiomas with Interferon-a. J Pediat Hematol/Oncol 1997; 19: 232-6.

8. Hancock BJ, St-Vil D, Luks FI, Di Lorenzo M, Blanchard H. Complications of lymphangiomas in children. J Pediatr Surg 1992; 27: 220-6.

9. Schmidt B, Schimpl G, Höllwarth ME. OK-432 therapy of lymphangiomas in children. Eur J Pediatr 1996; 155: 649-52.

10. Ogita $S$, Tsuto $T$, Tokiwa $K$, Takahashi $T$. Intracystic injection of OK-432: A new sclerosing therapy for cystic higroma in children. Br J Surg 1987; 74: 690-1.

11. World Health Organization. WHO Handbook for reporting results of cancer Treatment. WHO Offset Publication $\mathrm{N}^{\circ} 48$. Geneva: WHO; 1979.

12. White CW, Wolf SJ, Korones DN, Sondheimer HM, Tosi MF, $\mathrm{Yu}$ A. Treatment of childhood angiomatous diseases with recombinant interferon alfa-2a. J Pediatr 1991; 118: 59-66.

13. Ricketts RR, Hatley RM, Corden BJ, Sabio H, Howell CG. Interferon-alpha-2a for the treatment of complex hemangiomas of infancy and childhood. Annals Surg 1994; 219: 605-14.

14. White CW, Sondheimer HM, Crouch EC, Wilson H, Fan LL. Treatment of pulmonary hemangiomatosis with recombinant interferon alpha-2a. N Engl J Med 1989; 320: 1197-200.

15. Maddalozzo J, Hughes A, Huang L, Um Y, Ludemann J, Crawford S. High angiogenic activity in cells isolated from cystic hygroma. Arch Otolaryngol Head Neck Surg 1999; 125: 45-8.

16. Wahrman JE, Honig PJ. Hemangiomas. Pediatr Rev 1994; 15 : 266-71.

17. Sadan N, Wolach B. Treatment of hemangiomas of infants with high doses of prednisone. J Pediatr 1996; 128: 141-6.

18. Neidhart JÁ, Roach RW. Successful treatment of skeletal hemangioma and Kasabach-Merrit syndrome with aminocaproic acid. Is fibrinolysis "defensive"? Am J Med 1982; 73: 434-8.

19. Ortel TL, Onorato JJ, Bedrosian CL, Kaufman RE. Antifibrinolytic therapy in the manangement of the Kasabach-Merrit syndrome. Am J Hematol 1988; 29: 44-8.

Endereço para correspondência:

Dr. Luiz Gonzaga Tone

HC-FMRP- USP - Av. Bandeirantes, 3.900

Ribeirão Preto - SP - CEP 14048-900

Fone: (16) 633.0136 - Fax: (16) 602.2700 